\title{
Unperturbed Chain Dimensions of Poly(4-methyl-pentene-1) in Theta Solvents
}

\author{
Shizuma Tani, Fumiyuki Hamada, and Akio Nakajima* \\ Department of Polymer Chemistry, Kyoto University, \\ Sakyo-ku, Kyoto 606, Japan. \\ (Received February 15, 1973)
}

\begin{abstract}
The unperturbed mean-square end-to-end distance $\left\langle R_{0}^{2}\right\rangle$ and its temperature variation $\mathrm{d} \ln \left\langle R_{0}^{2}\right\rangle / \mathrm{d} T$ for isotactic poly(4-methyl-pentene-1) have been estimated from limiting viscosity data in three theta solvents, i.e., diphenyl, diphenyl ether, and diphenyl methane. Measurements were made at the theta temperatures as determined from precipitation temperature measurements. The characteristic ratios, $\left\langle R_{0}^{2}\right\rangle / n l^{2}$, where $n$ is the number of bonds of length $l$ in the main chain, evaluated by assuming $\Phi=2.5 \times 10^{21}$, are 13.1 in diphenyl methane (at $\Theta=176.6^{\circ} \mathrm{C}$ ), 12.7 in diphenyl (at $\Theta=194.6^{\circ} \mathrm{C}$ ), and 13.2 in diphenyl ether (at $\Theta=210.0^{\circ} \mathrm{C}$ ). These values lead to the temperature coefficient $\mathrm{d} \ln \left\langle R_{0}^{2}\right\rangle / \mathrm{d} T \cong 0$. The results obtained were in support of the theoretical calculation reported by Abe.

KEY WORDS Poly(4-methyl-pentene-1) / Unperturbed Chain Dimensions / Precipitation Temperature / Phase Diagram /
\end{abstract}

The unperturbed chain dimension and its temperature variation for linear polyethylene and stereoregular poly- $\alpha$-olefins have been theoretically investigated by Nagai, ${ }^{1}$ Flory et al., ${ }^{2,3}$ and Abe. ${ }^{4}$ Experimentally, these quantities were estimated on linear polyethylene, ${ }^{5}$ iso-polypro-

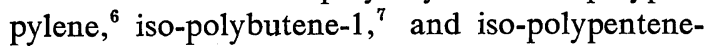
$1 .^{8}$ In particular, the experimental results on iso-polypropylene reported by $\mathrm{us}^{6}$ were supported by Abe's theoretical results ${ }^{4}$ on poly- $\alpha$ olefins having taken into account the effect of side chains attached to the $\alpha$-carbons. The side chains of poly(4-methyl-pentene-1) (P4MP1) are larger than those of polypropylene, and thus may effect the unperturbed chain dimensions more markedly in comparison with polypropylene.

The aim of this report is to determine the unperturbed chain dimensions in several theta solvents, and to compare the experimental results with the calculations given by Abe. ${ }^{4}$

\section{EXPERIMENTAL}

\section{Polymer Samples}

The monomer, 4-methyl-pentene-1, purified

\footnotetext{
* To whom correspondence should be addressed.
}

through molecular sieve, was polymerized ${ }^{9}$ for $3 \mathrm{hr}$ at $50^{\circ} \mathrm{C}$ with a Ziegler-Natta catalyst consisting of $\mathrm{Al}\left(\mathrm{C}_{2} \mathrm{H}_{5}\right)_{3} / \mathrm{TiCl}_{4}=2.6$ in $n$-heptane. The polymer obtained was extracted with boiling $n$-hexane to remove stereoirregular molecular species. About 50\% of the polymer remained after the extraction. This highly isotactic residual sample was dissolved in decalin containing 100-ppm $N, N^{\prime}$-di- $\beta$-naphtyl-p-phenyl diamine (a stabilizer to prevent chain degradation at elevated temperature). Then to obtain different molecular weights, the sample was separated into ten fractions by successive precipitations at $130^{\circ} \mathrm{C}$ by adding the non-solvent ethyl cellosolv. Five fractions chosen from these fractions were further purified by reprecipitation from xylene, and used for the experiments.

The isotacticity of these fractions was kindly investigated by Dr. Fujiwara of Kuraray Co. According to his estimation from NMR spectra, the isotacticity of the fractions is expected to be ca. 0.90-0.94.

\section{Viscosity Measurements}

The limiting viscosity numbers of the fractions were determined in decalin at $130^{\circ} \mathrm{C}$, and in the three theta solvents at their theta temperatures, i.e., in diphenyl methane at $176.6^{\circ} \mathrm{C}$, in diphenyl 
at $194.6^{\circ} \mathrm{C}$, and in diphenyl ether at $210.0^{\circ} \mathrm{C}$. Viscosity measurements were carried out with a modified Ubbelohde dilution viscometer manufactured by us. ${ }^{5}$ Decalin used as the solvent was a mixture of $\operatorname{cis}(70.2 \%)$-and $\operatorname{trans}(29.8 \%)$ decalin, and its density was 0.806 at $130^{\circ} \mathrm{C}$. For measurements at $130^{\circ} \mathrm{C}$ in decalin, $100-\mathrm{ppm}$ $N, N^{\prime}$-di- $\beta$-naphthyl- $p$-phenyl diamine was added to the solutions. For measurements at theta temperatures, the concentration of this stabilizer in solutions was about $0.2 \%$. Freshly prepared solutions containing the stabilizer were used for viscosity measurements so as to eliminate the possibility for chain scission in solutions at elevated temperature.

\section{Osmotic Pressure Measurements}

The number-average molecular weights of these five fractions were determined from the osmotic pressure in tetralin at $130^{\circ} \mathrm{C}$. A highspeed membrane osmometer equipped with a UCF-super dense semipermeable membrane was used for the measurements. Polymer solutions in tetralin were stabilized with $0.2-\%$ 2,6-di-tertbutyl-p-cresol to prevent chain degradation. The stabilizer was also added to the solvent side of the apparatus.

\section{Measurements of Precipitation Temperature}

Diphenyl methane, diphenyl, and diphenyl ether were used as the theta solvents. The polymer fraction was added to the diluent containing 0.5-\% 2,6-di-tert-butyl-p-cresol, and the mixture was sealed in a glass tube under a nitrogen atmosphere. The contents were heated to dissolve the polymer completely, then slowly cooled down to determine the precipitation temperature $T_{\mathrm{p}}$ by observing the appearance of turbidity. The critical precipitation temperature $T_{\mathrm{c}}$ for each fraction was determined as the maximum temperature of the precipitation temperature $v s$. polymer concentration curve.

\section{RESULTS AND DISCUSSION}

\section{Molecular Weight-Limiting Viscosity Number Relationship}

The molecular weights obtained from the osmotic pressures and the limiting viscosity numbers in decalin at $130^{\circ} \mathrm{C}$ were plotted on a double logarithmic scale in Figure 1. A straight

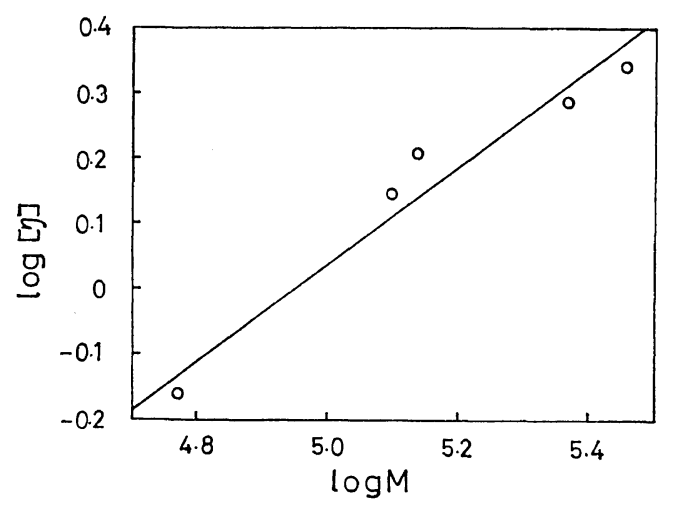

Figure 1. Limiting viscosity number-molecular weight relationship for poly(4-methyl-pentene-1) in decalin at $130^{\circ} \mathrm{C}$.

line obtained by the least-squares method leads to the equation:

$[\eta]=1.95 \times 10^{-4} M^{0.75}$, in Decalin at $130^{\circ} \mathrm{C}(1)$

\section{Determination of Theta Temperature}

In Figures 2-4, the precipitation temperatures, $T_{\mathrm{p}}$ are plotted against the volume fractions, $v_{2}$, of polymer. The maximum point of the curve corresponds to the critical temperature, $T_{\mathrm{c}}$. The reciprocal critical temperatures, $1 / T_{\mathrm{c}}$, were plotted against the reciprocals of the root molecular weights, $1 / M^{1 / 2}$, for the three solvent systems in Figure 5. From this plot the theta temperatures $\Theta$ for these solvent systems were determined in accordance with the well-known Flory-Schulz equation: ${ }^{10}$

$$
\begin{aligned}
1 / T_{\mathrm{c}} & =1 / \Theta\left(1+b / M^{1 / 2}\right), \\
b & =\left(V_{\mathrm{s}} / \tilde{v}\right)^{1 / 2} / \psi_{1}
\end{aligned}
$$

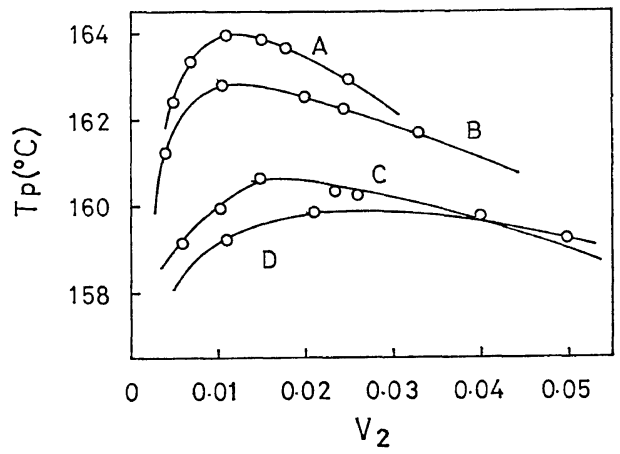

Figure 2. Phase diagram for four poly(4-methylpentene-1) fractions in diphenyl methane. Molecular weights of A, B, C, and D are 228600, 190000, 169600 , and 137400 , respectively. 


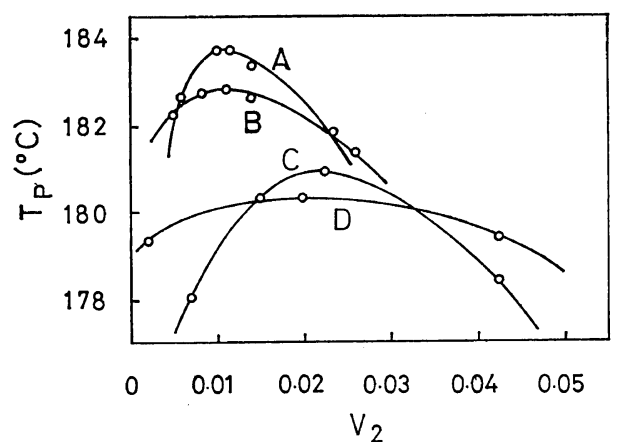

Figure 3. Phase diagram for four poly(4-methylpentene-1) fractions in diphenyl. Molecular weights of fractions are given in Figure 2.

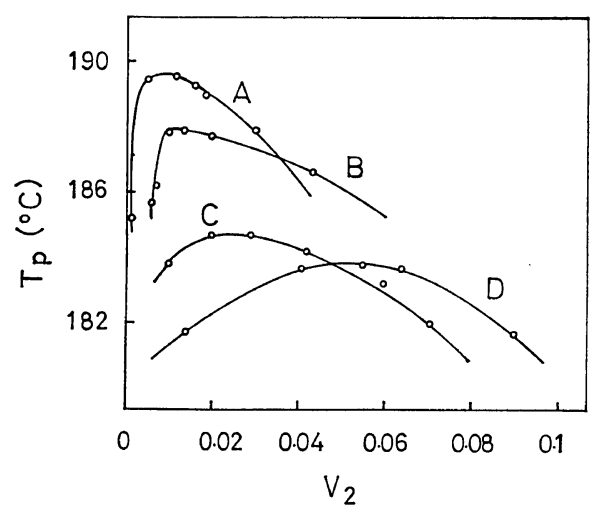

Figure 4. Phase diagram for four poly(4-methylpentene-1) fractions in diphenyl ether. Molecular weights of fractions are given in Figure 2.

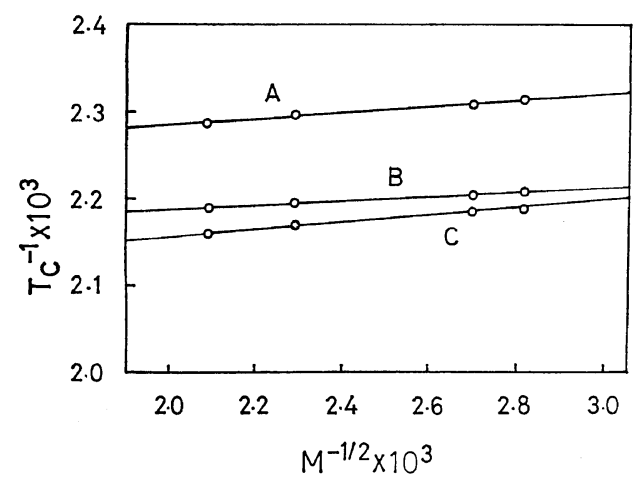

Figure 5. Plots of the reciprocal critical temperature against the reciprocal of the root molecular weight for poly(4-methyl-pentene-1) in diphenyl methane (A), diphenyl (B), and diphenyl ether (C). where, $V_{\mathrm{s}}$ is the molar volume of the solvent, $\tilde{v}$ the partial specific volume of the polymer, and $\psi_{1}$ is the entropy parameter. The $\Theta$ values obtained were shown in Table I, together with the results on the chain dimensions.

\section{Limiting Viscosity Number at the Theta Tem- perature}

The limiting viscosity numbers at the theta temperature $[\eta]_{\theta}$ for P4MP1 fractions in diphenyl methane $\left(176.6^{\circ} \mathrm{C}\right)$, diphenyl $\left(194.6^{\circ} \mathrm{C}\right)$, and diphenyl ether $\left(210.0^{\circ} \mathrm{C}\right)$ are plotted against the molecular weight $M$ on a double logarithmic scale in Figures 6, 7, and 8, respectively. The polymer concentration $c$ in $\mathrm{g} / \mathrm{d} l$ units was determined from the weight concentrations by

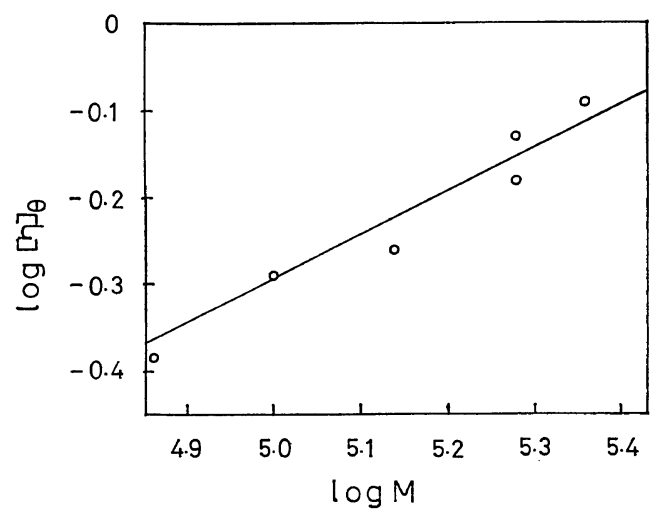

Figure 6. Double logarithmic plot of the limiting viscosity number in diphenyl methane at its theta temperature $\left(176.6^{\circ} \mathrm{C}\right)$ against the molecular weight for poly(4-methyl-pentene-1).

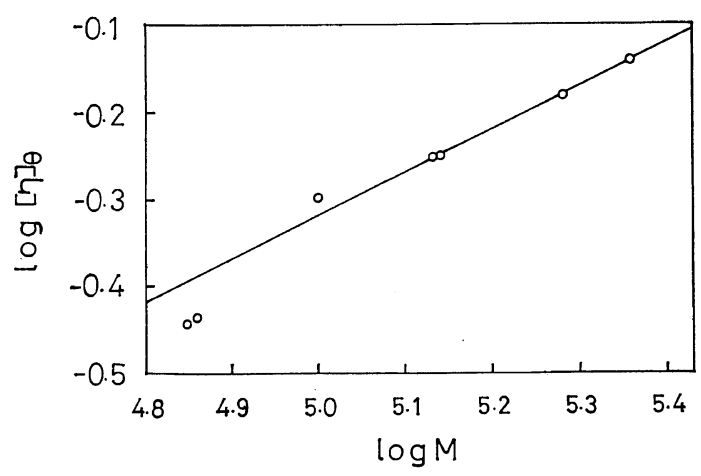

Figure 7. Double logarithmic plot of the limiting viscosity number in diphenyl at its theta temperature $\left(194.6^{\circ} \mathrm{C}\right)$ against the molecular weight for poly(4-methyl-pentene-1). 
Unperturbed Chain Dimensions of Poly(4-methyl-pentene-1) in Theta Solvents

Table I. Characteristic ratios of P4MP1 in three theta solvents

\begin{tabular}{lcccc}
\hline Solvent & $\Theta$ temp, ${ }^{\circ} \mathrm{C}$ & $K_{\theta} \times 10^{3}$ & $\left\langle R_{0}^{2}\right\rangle / n l^{2}$ & $\mathrm{~d} \ln K_{\theta} / \mathrm{d} T$ \\
\hline Diphenyl methane & 176.6 & 1.60 & 13.1 & \\
Diphenyl & 194.6 & 1.52 & 12.7 & $\cong 0$ \\
Diphenyl ether & 210.0 & 1.58 & 13.2 & \\
\hline
\end{tabular}

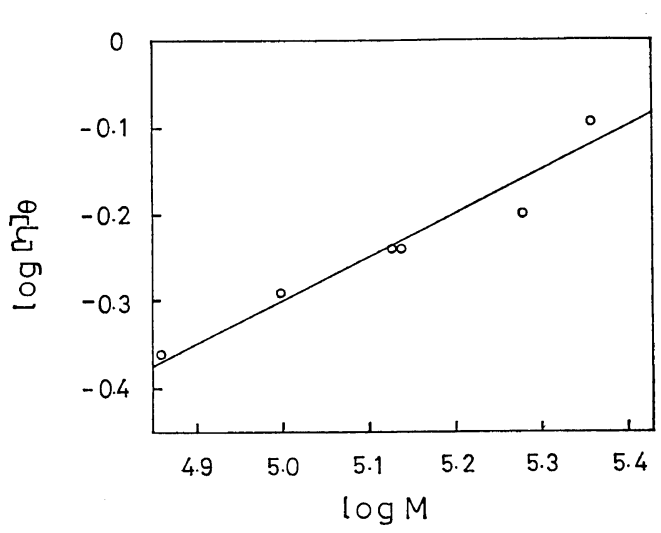

Figure 8. Double logarithmic plot of the limiting viscosity number in diphenyl ether at its theta temperature $\left(210.0^{\circ} \mathrm{C}\right)$ against the molecular weight for poly(4-methyl-pentene-1).

using the densities of the polymer and the solvents as a function of temperature. The density of polymer as a function of temperature was taken from Rånby's results. ${ }^{11}$ With respect to solvents, densities in $\mathrm{g} / \mathrm{m} l$ units were determined as a function of temperature. They were: $\rho($ diphenyl methane $)=1.026-8.18 \times 10^{-4} \mathrm{~T}, \rho(\mathrm{di}-$ phenyl $)=1.056-8.60 \times 10^{-4} T$, and $\rho$ (diphenyl ether) $=1.097-9.13 \times 10^{-4} T$, in which $T$ is in ${ }^{\circ} \mathrm{C}$ units.

The experimental points shown in Figures 6-8 are approximately fitted by a straight line with a slope of $1 / 2$ as predicted theoretically by

$$
[\eta]_{\theta}=K_{\theta} M^{0.5}
$$

The values of $K_{\theta}$ obtained were given in Table I. Unperturbed Chain Dimension and Its Temperature Variation

The $K_{\theta}$ is related to the unperturbed meansquare end-to-end distance $\left\langle R_{0}^{2}\right\rangle$ by

$$
K_{\theta}=\Phi\left[\left\langle R_{0}^{2}\right\rangle / M\right]^{3 / 2}
$$

where $\Phi$ is a constant. Accordingly, the characteristic ratio $\left\langle R_{0}^{2}\right\rangle / n l^{2}$ is written as

$$
\left\langle R_{0}^{2}\right\rangle / n l^{2}=\left[K_{\theta} / \Phi\right]^{2 / 3} M / n l^{2}
$$

in which $n$ and $l$ are the number of $\mathrm{C}-\mathrm{C}$ bonds in a chain and the length of a $\mathrm{C}-\mathrm{C}$ bond, respectively. For P4MP1, $M / n=42$ and $l=1.54 \AA$. The characteristic ratio calculated by using the value $\Phi=2.5 \times 10^{21}$ is given in Table $\mathrm{I}$.

The temperature variation of the unperturbed chain dimension is given by

$$
\frac{\mathrm{d} \ln \left\langle R_{0}^{2}\right\rangle}{\mathrm{d} T}=\frac{2}{3} \frac{\mathrm{d} \ln K_{\theta}}{\mathrm{d} T}
$$

From the values of $K_{\theta}$ for the three solvents at their theta temperatures, we obtain $\mathrm{d} \ln \left\langle R_{0}^{2}\right\rangle /$ $\mathrm{d} T \cong 0$.

The unperturbed mean-square end-to-end distance $\left\langle R_{0}^{2}\right\rangle$ of a chain molecule composed of $n$ $\mathrm{C}-\mathrm{C}$ bonds is mathematically represented by the following equation.

$$
\left\langle R_{0}^{2}\right\rangle=n l^{2}+2 \sum_{i<j}\left\langle\boldsymbol{l}_{i} \cdot \boldsymbol{l}_{j}\right\rangle
$$

where, $\boldsymbol{l}_{i}$ is the $i$ th $\mathrm{C}-\mathrm{C}$ bond vector, $l$ the bond length of a $\mathrm{C}-\mathrm{C}$ bond, and $\left\langle\boldsymbol{l}_{i} \cdot \boldsymbol{l}_{j}\right\rangle$ the average of the scalar product $\boldsymbol{l}_{i} \cdot \boldsymbol{l}_{j}$ over all available chain conformations.

Conformation calculations for P4MP1 were developed by $\mathrm{Abe}^{4}$ in his report on the unperturbed chain dimensions of poly- $\alpha$-olefins, $\mathrm{H}-\left[\mathrm{CH}_{2}-\mathrm{CHR}\right]_{\bar{n} / 2} \mathrm{CH}_{3}$. By taking into consideration the effect of the side chain $(-\mathrm{R})$ on the backbone chain conformations, i.e., the four-bond interactions due to side chain configuration, Abe introduced the statistical weight factors $\tau^{*}$ and $\omega^{\prime \prime}$ respectively for the $t t$ and $g^{+} g^{-}$(or $g^{-} g^{+}$)-conformations ( $t$ and $g^{ \pm}$denote trans and gauche conformations, respectively) of the adjoining skeletal bonds $i-1$ and $i$ jointed by the $\mathrm{C}$ atom of the CHR residue. These factors $\tau^{*}$ and $\omega^{\prime \prime}$. were normalized to unity for the stable helical conformation, and related to the conformational energies $E_{\tau^{*}}$ and $E_{\omega^{\prime \prime}}$ by $E_{\tau^{*}}=-R T \ln \tau^{*}$, and $E_{\omega^{\prime \prime}}=-R T \ln \omega^{\prime \prime}$, respectively. Abe assigned the following values for 
polypropylene $\left(\mathrm{R}=\mathrm{CH}_{3}\right)$, poly-butene-1 $\left(\mathrm{R}=\mathrm{CH}_{2}\right.$. $\left.\mathrm{CH}_{3}\right)$, and poly(4-methyl-pentene-1) $\left(\mathrm{R}=\mathrm{CH}_{2}\right.$. $\left.\mathrm{CH}\left(\mathrm{CH}_{3}\right)_{2}\right)$.

$\begin{array}{llc}\quad \mathrm{R} & E_{\tau^{*}} / R T & E_{\omega^{\prime \prime}} / R T \\ \mathrm{CH}_{3} & 0 & 4 \\ \mathrm{CH}_{2} \cdot \mathrm{CH}_{3} & 1.4 & 5 \\ \mathrm{CH}_{2} \cdot \mathrm{CH}\left(\mathrm{CH}_{3}\right)_{2} & 5 & 5\end{array}$

On the basis of the statistical weight matrix expressions for the stereochemical arrangements, $\mathrm{Abe}^{4}$ used eq 7 to carry out a Monte Carlo calculation on the unperturbed chain dimensions of a 100 monomer units P4MP1 Monte Carlo chain, which was generated in a computer as a function of stereoregularity $p_{r}$, wherein $p_{r}$ de-

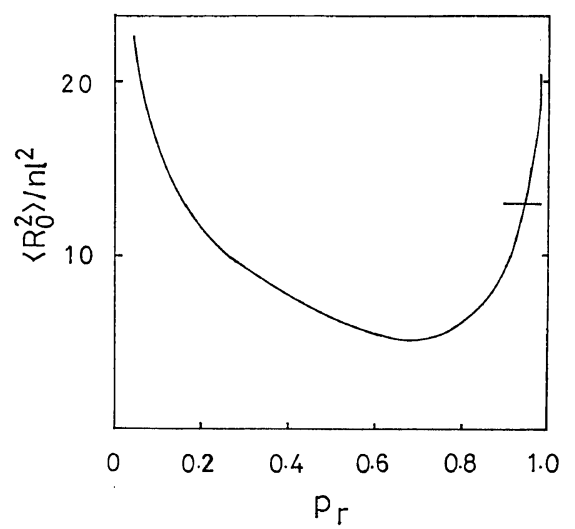

Figure 9. Characteristic ratio against isotacticity $p_{\mathrm{r}}$ for poly(4-methyl-pentene-1). The experimentally obtained value is shown by a horizontal bar. The curve was cited from Abe's paper. ${ }^{4}$

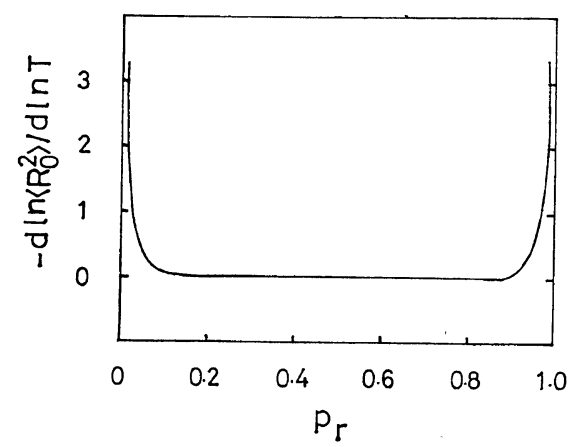

Figure 10. Temperature coefficient of the characteristic ratio as a function of isotacticity for poly(4-methyl-pentene-1), cited from Abe's paper.4 notes the probability of an isotactic dyad placement.

The curves obtained are reproduced in Figures 9 and 10. Our experimental value, $\left\langle R_{0}^{2}\right\rangle / n l^{2} \cong$ 13 , is in agreement with the theoretical value at $p_{\mathrm{r}}=c a$. 0.94. This value of $p_{\mathrm{r}}$ does not conflict with the isotacticity $p_{\mathrm{r}}=0.90-0.94$ estimated for our samples from NMR spectra. From Figure 10, it is pointed out that the experimentally obtained temperature variation $\mathrm{d} \ln \left\langle R_{0}^{2}\right\rangle /$ $\mathrm{d} T \cong 0$ is also supported by the calculation.

The final point to add is that P4MP1 is not dissolved in any solvent at temperatures below ca. $100^{\circ} \mathrm{C}$, hence various measurements with solutions had to be carried out at rather high temperatures and, at the same time, in the presence of the stabilizer to prevent chain degradation. Some scatters of data points seems to have been unavoidable.

Acknowledgment. The authors wish to express their sincere thanks to Dr. Y. Fujiwara of Kuraray Co. for the NMR investigation on the isotacticity of the samples, and to Dr. K. Yamaguchi of Showa Denko Co. for his valuable suggestions on the osmometry at elevated temperature.

\section{REFERENCES}

1. K. Nagai and T. Ishikawa, J. Chem. Phys., 37, 496 (1962).

2. P. J. Flory, J. E. Mark, and A. Abe, J. Amer. Chem. Soc., 88, 639 (1966).

3. A. Abe, R. L. Jernigan, and P. J. Flory, ibid., 88, 631 (1966).

4. A. Abe, Polymer J., 1, 232 (1970).

5. A. Nakajima, F. Hamada, and S. Hayashi, $J$. Polym. Sci., Part C, 15, 285 (1966).

6. A. Nakajima and A. Saijyo, ibid., Part A, 26, 735 (1968).

7. G. Moraglio, G. Gianotti, F. Zoppi, and U. Bonicelli, Eur. Polym. J., 7, 303 (1971).

8. G. Moraglio, and G. Gianotti, ibid., 5, 781 (1969).

9. A. Nakajima, S. Hayashi, T. Taka, and N. Utsumi, Koll-Z. Z. Polym. 234, 1097 (1969).

10. A. R. Shultz and P. J. Flory, J. Amer. Chem. Soc., 74, 4760 (1952).

11. J. H. Griffith and B. G. Ranby, J. Polym. Sci., 44, 369 (1960). 